Бутома Б.Г.', ${ }^{1,}$ Коцюбинский А.П. ${ }^{1}$

${ }^{1}$ Национальный медицинский исследовательский центр психиатрии и неврологии имени В.М. Бехтерева, Санкт-Петербург, Россия

${ }^{2}$ Санкт-Петербургский государственный университет, Санкт-Петербург, Россия

Butoma B. ${ }^{1,2}$, Kotsyubinsky A. ${ }^{1}$

${ }^{1}$ Bekhterev National Research Medical Centre for Psychiatry and Neurology, Saint-Petersburg, Russia

${ }^{2}$ Saint-Petersburg State University, Saint-Petersburg, Russia

\title{
Эффективность телесно-ориентированных
} методов психотерапии

\section{в персонализированном лечении пациентов с аутохтонными психическими расстройствами}

\author{
The Effectiveness of Body-Oriented Psychotherapy Methods \\ in Personalized Treatment of Patients with Autochthonous Mental \\ Disorders
}

Резюме

В статье приведен краткий обзор телесно-ориентированных методов психотерапии, используемых как в нашей стране, так и за рубежом. Авторы статьи, рассматривая человека в единстве его психосоциального и биологического функционирования, подчеркивают, что телесно-ориентированная психотерапия расширяет возможности общего психотерапевтического воздействия, помогая пациенту с помощью двигательных психотехник более точно распознать и вербализовать собственные эмоции. Это позволяет ему пережить заново свой прошлый эмоциональный опыт, способствуя тем самым расширению сферы самосознания и формированию более эмоционально благоприятного отношения к себе. Авторы статьи условно разделяют используемые в клинике многочисленные методы телесно-ориентированной психотерапии на 3 категории, позволяющие зарубежным специалистам рассматривать ее в качестве зонтичного метода: 1) методы психоаналитического направления; 2) методы поведенческого направления; 3) методы опытного направления. Авторами предлагается интегративный телесно-ориентированный метод, разработанный в отделении биопсихосоциальной реабилитации психически больных Национального медицинского исследовательского центра психиатрии и неврологии имени В.М. Бехтерева, показана эффективность этого варианта групповой психотерапии.

Ключевые слова: телесная терапия; телесно-ориентированная психотерапия; методы телесной психотерапии психоаналитического направления; методы телесно-ориентированной психотерапии, относящиеся к поведенческому направлению; методы телесно-ориентированной психотерапии опытного направления; интегративный телесно-ориентированный метод психотерапии; персонализированная терапия; невротические расстройства; депрессивные расстройства; тревожные расстройства; аутохтонные психические расстройства. 


\section{Abstract}

The article provides a brief overview of body-oriented methods of psychotherapy used both in our country and abroad. The authors of the article, considering a person in the unity of his psychosocial and biological functioning, emphasize that body-oriented psychotherapy expands the possibilities of general psychotherapeutic influence, helping the patient using motor psychotechnics for more accurately recognize and verbalize their own emotions. This allows him to relive his past emotional experiences, thereby contributing to the expansion of the sphere of self-awareness and the formation of a more emotionally favorable attitude towards himself. The authors of the article conditionally divide into 3 categories the numerous methods of body-oriented psychotherapy used in the clinic, which allow foreign specialists to consider this method as «umbrella»: 1) psychoanalytic direction; 2) behavioral direction; 3) experimental direction. The authors propose an integrative body-oriented method developed in the Biopsychosocial Therapy Department of V.M. Bekhterev National Research Medical Center for Psychiatry and Neurology, and show the effectiveness of this method of group psychotherapy.

Keywords: body therapy, body-oriented psychotherapy, methods of body-oriented psychotherapy of psychoanalytic direction, methods of body-oriented psychotherapy related to behavioral direction, methods of body-oriented psychotherapy experienced direction, integrative bodyoriented psychotherapy method, personalized therapy, neurotic disorders, depressive disorder, anxiety disorder, autochthonous mental disorders.

Современный период развития науки характеризуется стремлением к пониманию человеческого организма как целостной системы. С этой точки зрения телесно-ориентированные психотехнологии находят свою реализацию в пространстве интеграции тела и разума. Акцент при этом делается на знакомстве человека как с телом (и расширении тем самым сферы осознания глубоких ощущений организма), так и с разумом (осознании того, как потребности, желания и чувства кодируются в разных телесных состояниях), что в совокупности позволяет преодолеть барьер между физическим и психическим и способствовать обучению индивидуума умению разрешать интрапсихические конфликты [1].

Практика телесно-ориентированных методов психотерапии, осуществляемая как в нашей стране, так и за рубежом, представляет собой достаточно рыхлое гетерогенное поле. В иностранной литературе делались попытки дифференциации этого гетерогенного поля и выделения в качестве содержательно самостоятельных таких направлений, как телесная психотерапия (body psychotherapy BP), телесно-ориентированная психотерапия (body-oriented psychotherapy - BOP) и телесная терапия (body-therapy - BT). В то же время F. Rohricht [2], сравнивая определения телесно-ориентированных технологий, приводимые различными авторами, а также представленные на веб-сайтах европейских и американских ассоциаций телесной терапии (Европейская ассоциация телесной психотерапии (European Association of Body Psychotherapy - EABP); Американская ассоциация телесной психотерапии (United States Association of Body Psychotherapy-USABP); Европейский форум психомоторики (European 
Forum of Psychomotricity) и Английская ассоциация танцевально-двигательной терапии (Association of Dance Therapy - ADMT-UK)), приходит к выводу, что они очень мало отличаются друг от друга, в связи с чем предлагает использовать единый термин «телесно-ориентированная психотерапия» (body-oriented psychotherapy - BOP), который следует понимать как зонтичный термин. При этом важным является указание на потенциально уникальные возможности телесно-ориентированной психотерапии в системе лечения психических расстройств [2-4], подразумевая при этом многообразие используемых методов, которые можно условно разделить на 3 следующие категории [5]:

І. Методы психоаналитического направления, которые, с точки зрения авторов, влияют на такой компонент самосознания, как самопонимание. Предполагается, что в процессе работы с позой и мышечными зажимами пациента, а именно воздействуя на физический аспект его сопротивления и оказывая тем самым влияние на защитные механизмы индивидуума, можно преодолеть психическое сопротивление в целом и тем самым способствовать осознанию вытесняемых потребностей и конфликтов личности. К этой группе методов можно отнести: 1) характерологический анализ Вильгельма Райха (Wilhelm Reich, 1897-1957) [1, 6, 7]; 2) биоэнергетический анализ Александра Лоуэна (Alexander Lowen, 1910-2008) [1, 7-9]; 3) биосинтез Давида Боаделлы (David Boadella, p.1931) [10]; 4) бодинамический анализ (бодинамика) Лизбет Марчер (Lisbeth Marcher) $[8,11]$; 5) тимопрактику, или концентрированную двигательную терапию [12-14]; 6) метод Джона Пьерракоса (John Pierrakos, 1921-2001) [15, 16]. В западной литературе метод обозначается термином core-energetics («стержневая энергетика») [15, 16], основан на интеграции в понимании энергетических систем организма и духовной природы людей.

II. Методы телесно-ориентированной психотерапии, относящиеся к поведенческому направлению, которые ориентированы на представление об образе действий человека и направлены на замену неадаптивных форм поведения на адаптивные. По мнению приверженцев этого направления, неадаптивные формы поведения можно корректировать, обучая пациентов эффективному экономичному способу действия. Особенно это важно при снижении энергетического потенциала у психически больных. (реди этих методов можно выделить: 1) метод Моше Фельденкрайза (Moshe Feldenkrais, 1904-1984) [1, 7, 17, 18]; 2) метод Фредерика Матиаса Александера (Frederick Matthias Alexander, 18691955) $[7,19-21]$.

III. Методы опытного направления, нацеленные на изменение отношения пациента к себе, принятие себя и своего тела, что позволяет индивидууму повысить уровень сенсорной чувствительности и структурирования перцепции, а также уровень самоприятия: 1) метод чувственного осознания Шарлотты Селвер (Charlotte Selver, 1901-2003) [18, 22, 23]; 2) метод танатотерапии В.Ю. Баскакова [7, 18, 24], включающий приемы установления или восстановления гипотетического контакта человека с различными видами смерти и умирания, основан на модели тотального расслабления; 3) базовая терапия осознания тела (в некоторых источниках - культивация телесного осознания) (Basic Body Awareness Therapy - BBAT) [25-27], разработанная в 70-х гг. 
В настоящее время можно выделить еще одну группу методов телесно-ориентированной психотерапии, которая получила распространение в качестве комбинированных подходов. В этой группе можно указать следующее методы: 1) рольфинг, или метод структурной интеграции, разработанный Идой Полин Рольф (Ida Pauline Rolf, 1896-1979) и основанный на принципах характерологического анализа В. Райха в соединении с системой мануальной терапии с элементами кинезиотерапии [1, 7, 28, 29]; 2) метод Аллена Дарбонна (Allen Darbonne) [30-32] - соединение рольфинга, биоэнергетики и гештальт-терапии; 3) метод Иланы Рубенфельд (llana Rubenfeld) [33] - комбинацию метода Ф.М. Александера, метода М. Фельденкрайза и гештальт-терапии; 4) первичную терапию Артура Янова (Arthur Janov, 1924-2017) [1, 34] - комбинацию райхианской терапии и гештальт-терапии; 5) систему эвтонии, созданную Гердой Александер (Gerda Alexander, 1908-1994) [35, 36], которая напоминает технику Ф.М. Александера и предназначена для восстановления мышечного тонуса путем облегчения потока заблокированной энергии через коллективное бессознательное Юнга; 6) метод Хакоми (Hakomi). Термин «хакоми» (Hakomi) гносеологически связан с языком индейского племени хопи и может быть переведен как «кто ты в этом множестве миров?» Данный метод представляет собой синтез из многих направлений психотерапии (система Райха, биоэнергетика, гештальт-терапия, психомоторная терапия, метод Фельденкрайза, эриксоновский гипноз и нейролингвистическое программирование), во многом созвучен буддизму и даосизму с их мягкостью, состраданием, осознанностью и следованием естественной природе вещей, а также с определенным влиянием общей теории систем, которая привнесла в него идею уважения мудрости каждой личности как живой самоорганизующейся системы [37].

В целом методы телесной терапии, безусловно, отвечают требованиям целостного терапевтического подхода, поскольку их авторы рассматривают человека как единое функционирующее целое, сплав тела и психики, в котором изменения в одной области сопровождаются изменениями в другой. При таком понимании телесно-ориентированная психотерапия помогает клиенту с помощью двигательных психотехник более точно распознать и вербализовать собственные эмоции, пережить заново свой прошлый эмоциональный опыт, тем самым способствуя расширению сферы самосознания и формированию более эмоционально благоприятного отношения к себе. Помимо этого, она вносит определенный вклад в дальнейшее развитие наших представлений о механизмах лечебного действия психотерапии, поскольку изменения, происходящие с пациентом и являющиеся конечной целью психотерапевтического воздействия, относятся к различным уровням функционирования организма и личности [5].

Следует отметить, что телесно-ориентированные методы первоначально разрабатывались и применялись в основном для работы с невротическими пациентами [38] или с пациентами, имеющими психосоматические проблемы [39]. Лишь позднее телесно-ориентированная психотерапия стала успешно применяться у пациентов с психогенными тревожными и депрессивными расстройствами $[1,40]$.

Однако предлагаемые при этом техники, ориентированные на такие категории пациентов, являются часто психологически 
конфронтационными и активно действующими, вызывающими бурное эмоциональное отреагирование, что не совсем применимо к пациентам, страдающим эндогенными психическими расстройствами [5].

В связи с этим в отделении биопсихосоциальной реабилитации психически больных НМИЦ ПН им. В.М. Бехтерева был разработан вариант групповой психотерапии, который определен как интегративный телесно-ориентированный метод.

Оригинальность предлагаемого метода, как думается, заключается $[5,41,42]: 1)$ в теоретическом обосновании в виде представления о биопсихосоциальной сущности психических расстройств [43] с привлечением принципов этологического анализа поведения человека [44]; 2) интеграции известных методов телесной психотерапии, а именно: а) характерологического анализа В. Райха [1, 6, 7]; б) биоэнергетического анализа А. Лоуэна [1, 7-9]; в) метода М. Фельденкрайза [1, 8, 17, 18]; г) метода Ф.М. Александера $[1,7,19-21]$; д) элементов танатотерапии В.Ю. Баскакова («Театр прикосновений» и «Телесная гомеопатия») [7, $18,24]$ и ряда других; 3) интеграции методов телесно-ориентированной психотерапии и отдельных положений когнитивно-поведенческой коррекции, что обусловлено общностью фундирующих эти психотерапевтические направления этиопатогенетических представлений и теоретических основ; 4) рассмотрении при изучении эффективности данного метода приспособительного поведения человека как целостного показателя его психосоциального восстановления [5, 41, 42].

Представление о биопсихосоциальной сущности психических расстройств рассматривалось уже в трудах В.М. Бехтерева, П.Б. Ганнушкина и Д.С. Мелехова, но терминологически закрепилось в работах G.L. Engel (1982) [43] и способствовало появлению интереса исследователей не только к феноменологическим особенностям заболевания, но и к изучению психологических особенностей пациентов и характера их социального приспособления.

Этологические принципы, на которых основан интегративный метод телесной терапии, заключаются в следующем. Проблема понимания человека человеком уходит своими корнями в более общую проблему познания окружающего мира биологическими системами. Активное общение различных особей между собой является базовым атрибутом всех живучих организмов. Цель этого общения - обмен информацией как между представителями своего вида (внутривидовое общение), так и между особями другого вида (межвидовое общение). Главным средством коммуникации является естественный язык. Артикулированная речь при этом - основной канал для обмена информацией у вида Homo sapiens [44]. Однако пропозициональный язык не является исчерпывающим средством общения. Не менее важную роль играет праязык, включающий в себя выразительные движения и так называемую фонацию, значение которых модифицирует значение пропозициональных сообщений, а иногда играет и самостоятельную роль в процессе общения. В связи с этим под языком общения понимается вся совокупность жестов и реакций, в том числе и вокальных, служащая источником информации при взаимодействии людей друг с другом [45]. Так, С.И. Розум [45, 46] считает, что общение является одним из важнейших средовых условий, в которых происходит как формирование, так и функционирование личности. 
Известно, что многие психические заболевания характеризуются изменением или утратой социальных (межличностных) контактов, тогда как витальные биологические функции сохраняются и иногда по мере развития болезни становятся преобладающими. Развитие аутохтонных психических расстройств не только приводит к нарушению мимики, поз, жестов, т. е. невербального поведения в целом, но и способствует неправильному восприятию и пониманию невербальных аспектов коммуникации между индивидуумами (как следствие - нарушение когнитивных функций), что затрудняет адекватное функционирование личности в окружающей среде. Постулирование такой связи позволяет рассматривать этологические характеристики коммуникативного процесса как одно из проявлений нарушений когнитивных функций при шизофрении. В связи с этим этологический аспект в процессе улучшения коммуникаций (установление основанной на взаимопонимании информационной связи между людьми при их непосредственном взаимодействии) становится чрезвычайно необходимым при развитии психического расстройства, так как, являясь одним из главных компонентов общения [45], способствует социальной адаптации пациента.

Разработка нами метода интегративной телесно-ориентированной психотерапии и способа его применения была вызвана необходимостью интенсификации процесса терапии пациентов с эндогенными психическими заболеваниями, повышением их адаптивного потенциала, восстановлением когнитивных функций. Сконструированный интегративный метод телесно-ориентированной психотерапии, воздействуя как на механизмы психологической защиты $[1,47]$, так и на выработку адаптивных копинг-стратегий $[5,48]$, включает в себя специально подобранные методики и направлен на обеспечение безопасных условий проведения психотерапии, необходимых для пациентов с аутохтонными психическими расстройствами. Этот метод помогает пациентам пережить свой опыт как взаимосвязь психического и телесного, принять себя в этом качестве и тем самым получить возможность для улучшения своего функционирования в социуме. Новым и оригинальным, на наш взгляд, является введение в методику проведения телесно-ориентированной психотерапии элементов арт-анализа $[49,50]$. В ходе занятий пациенты выполняли проективные рисуночные техники. Рисуночный тест, как правило, проводился на первом и на одном из последних занятий. Пациенты, которые на рисунках в начале групповых занятий изображали только свое лицо или нечетко прорисовывали тело, после окончания групповой работы рисовали всю фигуру, причем изображая ее более пропорционально.

Методика проведения занятий, ориентированных на тело, включала 3 этапа, соответственно логике группового процесса, и подробно описана в ранее опубликованных работах [5, 48].

Интеграция методов телесно-ориентированной психотерапии и отдельных положений когнитивно-поведенческой коррекции происходила на различных этапах проведения данного метода. Если на первых этапах в основном использовался поведенческий тренинг, направленный на улучшение коммуникативных возможностей и создание безопасной и доверительной атмосферы в группе, то на последующих применялись и элементы, направленные на улучшение когнитивного 
функционирования пациентов. Все пациенты по мере посещения групповых занятий в индивидуальном порядке получали информацию о репертуаре своих психологических защит, стратегиях и тактиках поведения, социальной маске или персоне, идеальном, реальном, телесном «Я» и рекомендации к более адаптивному поведению и взаимодействию с окружающими. При необходимости пациенты получали индивидуальные консультации ведущих занятия психотерапевтов. Из самоотчетов пациентов следует, что они после окончания групповых занятий становились увереннее в себе; у них повышалась самооценка, появлялось уважение к своему телу, своим импульсам и желаниям, улучшалось настроение, уменьшалась тревога; у пациентов облегчалось общение, многие из них находили себе друга или подругу (вне группы) [5]. Таким образом, данные результаты подтверждают положение, высказанное У. Матураной (U. Maturana) [51, 52], о том, что понимание людьми друг друга зависит от степени сходства их когнитивных областей, т. е. областей их взаимодействий.

Сравнительная оценка эффективности метода интегративной телесно-ориентированной психотерапии проводилась в сравнении с группами поведенческой психотерапии. Результаты представлены в предшествующих публикациях [5, 48].

Описываемая интегративная методика телесной терапии позволяет решать многие типовые задачи определения наиболее уязвимых или опорных зон адаптации пациента к жизни с помощью индивидуальных или групповых занятий, а также поиска терапевтических путей воздействия на дезадаптивные сферы жизни личности, влияя на бессознательное, бережно работая с психологическими механизмами адаптации пациента (см. таблицу).

Метод интегративной телесно-ориентированной психотерапии показан к применению в реабилитационном процессе при терапии аутохтонных психических расстройств и необходимости решения задач реабилитационной работы по адаптации данной категории пациентов к жизни в здоровом обществе.

В настоящее время интегративный метод телесно-ориентированной психотерапии требует дальнейшего усовершенствования (как теоретических подходов, так и разработки дальнейших практических

\section{Средние показатели эффективности реабилитационной работы на примере группы пациентов с аутохтонными психическими расстройствами, страдающих обсессивно-фобической симптоматикой, повышенным мышечным и психическим напряжением}

Average values of the effectiveness of rehabilitation work on the example of a group of patients with autochthonous mental disorders, suffering from obsessive-phobic symptoms, increased muscle and mental stress

\begin{tabular}{|l|l|l|}
\hline \multirow{2}{*}{ Показатель } & \multicolumn{2}{|l|}{ Основания работы } \\
\cline { 2 - 3 } & $\begin{array}{l}\text { Психотерапевтическая работа } \\
\text { на основании субъективных } \\
\text { наблюдений и традиционных } \\
\text { методов (повед., динамич. } \\
\text { терапии, арт-терапии) }\end{array}$ & $\begin{array}{l}\text { Психотерапевтическая работа } \\
\text { на основании данных метода } \\
\text { интегративной телесно-ориен- } \\
\text { тированной психотерапии } \\
\text { (с элементами арт-анализа) }\end{array}$ \\
\hline $\begin{array}{l}\text { Средняя продолжительность } \\
\text { ремиссии (в месяцах) }\end{array}$ & 10 & 12 \\
\hline Процент трудовой реадаптации & 32 & 39 \\
\hline
\end{tabular}


рекомендаций) в целях улучшения личностно-ориентированной реабилитационной программы пациентов с аутохтонными психическими расстройствами.

В дальнейшем данный метод может быть рекомендован к адаптации в региональных медицинских учреждениях, в частности в таких как городские психиатрические больницы, психоневрологические диспансеры, дневные стационары и другие учреждения, оказывающие внебольничную помощь пациентам с аутохтонными психическими расстройствами.

Следует согласиться с рядом авторов, которые считают, что доказательная база эффективности телесно-ориентированной психотерапии в рамках клинической психотерапии недостаточно развита $[2,53,54]$. Поэтому в целях повышения клинической доказательности основ терапевтических процессов в телесно-ориентированной психотерапии, телесного осознавания и восприятия, эмоционального и мышечного компонента необходимо продолжать изучение взаимосвязи психотерапии, психофизиологии и нейробиологии $[2,54]$. При условии выполнения этих требований телесно-ориентированная психотерапия может стать одним из важных психотерапевтических методов в лечении психических расстройств наряду с другими господствующими направлениями психотерапии, такими как психодинамическое, когнитивно-поведенческое и гуманистическое [54].

Указанный подход совпадает и с определениями, которые даются различными европейскими и американскими ассоциациями, объединяющими специалистов, практикующих различные варианты телесноориентированых методик. Опуская незначительные различия, можно сказать, что этот метод представляет собой «...ветвь психотерапии с давней историей, основывающейся на холистическом подходе, где тело не только «сома». Оно не существует отдельно от психических функций, а представляет собой сложное функциональное единство когнитивных, эмоциональных и физических взаимодействий» [2] (EABP, www.eabp. org; USABP, www. usabp.org; EFP, www. psychomot.org).

Разработанный метод и способ применения интегративной телесно-ориентированной психотерапии оказали эффективное влияние на интенсификацию процесса терапии пациентов с аутохтонными психическими расстройствами, повышение адаптивно-компенсаторного потенциала и коррекцию нарушенных у них когнитивных функций [5].

Вклад авторов: все авторы принимали участие в разработке концепции статьи и написании текста. Окончательная версия рукописи была одобрена всеми авторами.

Authors' contribution: all authors took part in the development of the concept of the article and writing the text. The final version of the manuscript was approved by all authors.

Конфликт интересов. Авторы заявляют об отсутствии конфликтов интересов.

Conflicts of interest. The authors declare no conflicts of interest. 


\section{- ЛИТЕРАТУРA/REFERENCES}

1. Yudina I.I. (2013) Telesno-orientirovannaya psihoterapiya kak metod psihoterapevticheskoj korrekcii v rabote $s$ pacientami, stradayushchimi depressiej [Body-oriented psychotherapy as a method of psychological adjustment in working with depressed patient]. Sovremennaya terapiya v psihiatrii i nevrologii, no 3-4, pp. 47-55.

2. Rohricht F. (2009) Body oriented psychotherapy. The state of the art in empirical research and evidence-based practice: A clinical perspective. Body, Movement and Dance in Psychotherapy, vol. 4, no 2, pp. 135-156. doi: 10.1080/17432970902857263

3. Tarsha M.S., Park S., Totora S. (2020) Body-Centered Interventions for Psychopathological Conditions: A Review. Frontiers in Psychology, vol. 10. doi: 10.3389/fpsyg.2019.02907

4. Bloch Atefi A., Smith J. (2014) The effectiveness of body-oriented psychotherapy: A review of the literature. Melbourne: PACFA.

5. Butoma B.G., Aristova T.A. (2012) Primenenie telesno-orientirovannyh metodov psihoterapii v kompleksnom lechenii bol'nyh endogennymi psihicheskimi rasstrojstvami [Application of the bodywork psychotherapy in complex treatment of the patient with endogenic mental disorders]. V.M. Bekhterev Review of Psychiatry and Medical Psychology, no 3, pp. 80-87.

6. Reich W. (1989) Charakteranalyse. Technik und Grundlagen. Köln: Kiepenheuer \& Witsch.

7. Sergeeva L. (ed) (2000) Telesno-orientirovannaya psihoterapiya. Khrestomatiya [Body-oriented psychotherapy. Reader]. SPb: Prakticheskayapsihoterapiya. (in Russian)

8. Berezina-Orlova V. (2010) (ed. comp.) Telesnaya psihoterapiya. Bodinamika [Body psychotherapy. Bodynamics]. M.: AST. Moscow. (in Russian)

9. Lowen A. (1971) The Language of the Body. London: Collier Macmillan.

10. Boadella D. (1987) Lifestreams: An Introduction to Biosynthesis. England: Rutledge Kegan \& Paul.

11. Marcher L., Fich S. (2010) Body Encyclopedia: A Guide to the Psychological Functions of the Muscular System. Berkeley, California: North Atlantic Books.

12. Iljine V. (1910) Patsiyenty igrayut v teatr: put' istseleniya tela i razuma [Patients play theatre: a way of healing body and mind] (Quoted by H. Petzold (1973). Gestalttherapie und Psychodrama. H. Petzold (1973), Kassel: Nicol-Verlag). (in Russian)

13. Merleau-Ponty M. (2005) Phenomenology of Perception. London: Routledge.

14. Kamper D., Wulf C. (1981) Die Wiederkehr des Köpers. Berlin, Frankfurt am Main.

15. Pierrakos J.C. (1976) Human Energy Systems Theory: History and New Growth Perspectives. New York: Institute for the New Age of Man.

16. Pierrakos J.C (2005) Core Energetics, Developing the Capacity to Love and Heal. Core Evolution Publishing.

17. Feldenkrais M. (2007) Osoznavaniye cherez dvizheniye [Awareness through movement]. M.: Institut obshchegumanitarnykh issledovaniy. (in Russian)

18. Baskakov V. (2016) (ed. comp.) Khrestomatiya po telesno-oriyentirovano psikhoterapii i psikhotekhnike [Reader on body-oriented psychotherapy and psychotechnics]. M.: Institut obshchegumanitarnykh issledovaniy. (in Russian)

19. Alexander F.M. (1931) The Use of the Self. London: Methuen \& Co., LTD.

20. Staring J. (2015) F. M. Alexander, the Use of the Self, and a 1932 Book Review + Discussion in the Yorkshire Post: A Failure to Impact Medical Science. Case Studies Journal, vol. 4, iss. 10. pp. 26-43.

21. Brennan P. (1997) Metod Aleksandera. Zhizn', zdorov'ye, sila [The Alexander Method. Life, health, strength]. Kiev: Sofia. (in Russian)

22. Brooks Charles V.M. (1986) Sensory Awareness: Rediscovery of Experiencing Through the Workshops of Charlotte Selver, Great Neck. N.Y.: Felix Morrow Pub.

23. Yasyr A. Available at: http://telo.by/bodytherapy/metod_chuvstvennogo_soznavaniya_sharlotti_selver.

24. Baskakov V. (2002) Tanatoterapiya: teoreticheskiye osnovy i prakticheskoye primeneniye [Thanatotherapy: theoretical foundations and practical application]. M.: Institute of thanatotherapy. (in Russian)

25. Totton N. (ed.) (2005) New dimensions in body psychotherapy. Open University Press.

26. Skattebo Ulla-Britt (2005) Basic Body Awareness Therapy and Movement Harmony. Oslo: Oslo University College.

27. Probst M.L., Skjaerven (2017) Physiotherapy in Mental Health and Psychiatry. London: Elsevier.

28. Rolf I. (1977) Rolfing: The Integration of Human Structures, Boulder. CO: Rolf Institute.

29. Rolf I. (1989) Rolfing - Strukturelle Integration: Wandel und Gleichgewicht der Korperstruktur. Munchen: Hugendubel Verlag.

30. Darbonne A.R., Crisis (1986) A review of theory, practice and research. Inter J Psychiatry, vol. 6, no 5, pp. 371-379.

31. Kepner J.I. (2001) Touching gestalt body process psychotherapy. Gestalt Review, vol. 5, no 2, pp. 97-114. doi: 10.5325/gestaltreview.5.2.0097

32. Kepner J.I. (2013) Body Process: A Gestalt Approach to Working with the Body. New York.

33. Rubenfeld I. (1978) The Alexander technique and Gestalt therapy (Gestalt synergy): Changing approaches to the psychotherapies (eds. H.H. Grayson, C-Jamaica, Loew). New York: Spectrum Publication.

34. Janov A. (1972) The Primal Revolution. New York: Simon \& Schuster.

35. Rimskij S. (ed.) (2017) Mudrost 'tela. Putevoditel ' po metodam telesno-orientirovannoj terapii: antologiya [The wisdom of the body. A Guide to Body-Oriented Therapy: An Anthology]. Moscow: Institut obshhegumanitarny issledovany'. (in Russian)

36. Alexander G., Eutony (1986) The holistic discovery of the total person. New York: Felix Morrow Pub.

37. Kurtz R. (2007) Body-centered Psychotherapy: The Hakomi Method. Mendocino: LifeRhythm Publication.

38. Sergeeva L. (2009) Telesno-oriyentirovannyye metody $v$ sisteme psikhoterapii bol'nykh zatyazh-nymi nevrozami [Body-oriented methods in the system of psychotherapy of patients with lingering neuroses] (PhD Thesis). Saint-Petersburg.

39. Sandomirsky M. (2005) Psikhosomatika i telesnaya terapiya: prakticheskoye rukovodstvo [Psychosomatics and body therapy: a practical guide] Moscow: Nezavisimaya firma «Klass». (in Russian)

40. Yakovlev S. (2009) Gruppovaya integrativnaya telesno-oriyentirovannaya psikhoterapiya zatyazhnykh depressivnykh i trevozhnykh rasstroystv [Group integrative body-oriented psychotherapy of protracted depressive and anxiety disorders] (PhD Thesis). Moscow.

41. Kotsyubinskiy A.P., Guseva O.V., Yerichev A.N., Medvedem S.E., Butoma B.G., Stepanova A.V. (2015) Osobennosti psikhoterapii [Features of psychotherapy]. Autokhtonnyye nepsikhoticheskiye rasstroystva [Autochthonous non-psychotic disorders]. Saint-Petersbur: SpetsLit, pp. 332-339, 380-382.

42. Butoma B.G., Stepanova A.V. (2020) Telesno-oriyentirovannaya psikhoterapiya pri autokhtonnykh psikhicheskikh rasstroystvakh. Biopsikhosotsial'naya psikhiatriya. Rukovodstvo dlya vrachey [Body-oriented psychotherapy for autochthonous mental disorders. Biopsychosocial Psychiatry. Guidelines for doctors]. Moscow: SIMK, pp. 758-760.

43. Engel G.L. (1982) Biopsychosocial model and metical education: who are to be the teacher? N. Eng. Journ. Med., vol. 306, pp. 802-805

44. Klain Z. (2000) The ethological approach to the study of human behavior. Neuroendocrin. Letters, vol. 21, pp. 477- 448.

45. Rozum S.I. (1998) Kategoriya znacheniya, protsess kommunikatsii i kognitivnyye narusheniya pri shizofrenii [Meaning category, communication process and cognitive impairment in schizophrenia]. V.M. Bekhterev Review of Psychiatry and Medical Psychology, no 1, pp. 9-12. (in Russian) 
46. Rozum S.I. (2007) Psikhologiya sotsializatsii i sotsial'noy adaptatsii cheloveka [Psychology of socialization and social adaptation of a person]. SaintPetersburg: Rech'. (in Russian)

47. Levi T.S. (2019) Telesno-oriyentirovannaya psikhoterapiya: Spetsifika, vozmozhnosti, mekhanizmy vozdeystviya [Body-oriented psychotherapy: specifics, possibilities, mechanisms of influence]. Scientific works of the Moscow State University for the Humanities (electronic journal), no 3. Available at: http://journals.mosgu.ru/trudy/article/view/991 (accessed 23.06.21). doi: 10.17805/trudy. 2019. 3. 2

48. Aristova T.A., Butoma B.G, Kotsyubinskiy A.P., Akimenko M.A. (eds.) (2008) Primeneniye telesno-oriyentirovannykh metodov dlya lecheniya bol'nykh nervno-psikhicheskimi rasstroystvami: posobiye dlya vrachey i psikhologov [The application of body-oriented methods for the treatment of patients with neuropsychiatric disorders: a guide for doctors and psychologists]. Saint-Petersburg: St. Petersburg V. M. Bekhterev Research Psychoneurological Institute, pp. 1-54.

49. Kopytina A.I. (ed.) (2006) Art-terapiya - novyye gorizonty [Art therapy - new horizons]. Moscow: Kogito-Center. (in Russian).

50. Solokhina T.A., Yastrebova V.V., Mitikhin V.G., Tiumenkova G.V. (2019) Psikhosotsial'naya reabilitatsiya i psikhosotsial'noye lecheniye pri shizofrenii: tseli, etapy, otsenka effektivnosti [Psychosocial Rehabilitation and Psychosocial Treatment in Schizophrenia: Goals, Stages, Evaluation of the Effectiveness]. Psychiatry, vol. 17, no 3, pp. 62-74. (in Russian)

51. Maturana U. (1995) Biologiya poznaniya, Yazyk i intellekt [Biology of cognition. Language and intellect]. Moscow: Progress. (in Russian)

52. Maturana U., Varela F. (2001) Drevo poznaniya: Biologicheskiye korni chelovecheskogo ponimaniya [Tree of Knowledge: Biological Roots of Human Understanding]. Moscow: Progress-Tradition. (in Russian)

53. Matveeva V.V., Enin K.A. (2015) Vozmozhnosti primeneniya telesno-oriyentirovannoy psikho-terapii v korrektsii pogranichnykh psikhicheskikh rasstroystv $u$ lits opasnykh professiy [Possibilities of using body-oriented psychotherapy in the correction of borderline mental disorders in persons of hazardous professions]. Ekologiya. Zdorov'ye. Sport: Materialy VI Mezhdunarodnoy nauchno-prakticheskoy konferentsii [Ecology. Health. Sport: Materials of the VI International Scientific and Practical Conference]. Chita: ZabGU, pp. 238-242. (in Russian)

54. Matveeva V.V., Enin K.A. (2015) K voprosu ispol'zovaniya sovremennoy telesno-oriyentirovannoy psikhoterapii v meditsine i psikhologii [On the issue of using modern body-oriented psychotherapy in medicine and psychology]. Lichnost'v menyayushchemsya mire: zdorov'ye, adaptatsiya, razvitiye. Spetsial'nyy vypusk (electronic journal), pp. 241-245. Available at: www.humjournal.rzgmu.ru. (in Russian)

Подана/Submitted: 26.04 .2021

Принята/Accepted: 19.10 .2021

Контакты/Contacts: butbor08@gmail.ru 